\title{
LA RECUPERACIÓN DE LA ECONOMÍA CUBANA DESDE 1993: SU ESTRUCTURA, DESEMPEÑO Y POLÍTICA ECONÓMICA
}

\author{
Emily Morris*
}

\section{INTRODUCCIÓN}

Después de la caída del 35\% entre 1990 y 1993, la economía cubana ha entrado en un período de recuperación. El modesto crecimiento de $0.7 \%$ en 1994 , fue seguido por un $2.5 \%$ en 1995 y un $7.8 \%$ en 1996 . (Ver cuadro 1). El presente artículo al tiempo que examina la naturaleza de esta recuperación, también busca presentar algunas conclusiones acerca de su mantenimiento. La segunda parte, con la ayuda de los datos estadísticos disponibles, analiza la estructura de la producción y el comercio entre 1990 y 1996 a fin de precisar las principales causas del colapso de la economía cubana entre 1990 y 1993. El análisis estadístico también permitirá mostrar los factores que dieron inicio a la recuperación económica desde 1993. Las actuales limitaciones tanto de caracter doméstico como externo son el tema central de la sección 3. Se busca establecer hasta qué punto los factores externos contribuyeron a esta recuperación, lo que facilitaría además determinar hasta que grado la política gubernamental lo ha inhibido. En la sección 4 se ofrecen algunas conclusiones tentativas acerca del desempeño de la economía cubana hasta la fecha y se aventuran algunas proyecciones para los próximos años. de origen oficial, y aunque no es totalmente confiable, es a nuestro entender la más completa. Hasta 1989, las transacciones fueron calculadas de acuerdo con el sistema CAME, definido como el producto social global (PSG). En 1990, la publicación regular de los ingresos nacionales fue suspendida. Entre 1990 y 1994 la publicación de cifras estadísticas fue esporádica, concentrándose únicamente en algunos indicadores globales. A partir de 1995, el Banco Central empezó a producir un informe económico anual, haciendo uso de sistemas de medición económica aceptados internacionalmente, tales como el Producto Interno Bruto (PIB). Estas cifras son la fuente principal del presente estudio. Aunque los datos estadísticos no presentan distorsiones de caracter sistemático, es importante señalar que subsisten vacíos e inconsistencias notables.

El estudio analiza, en primer lugar, la producción doméstica sector por sector entre 1990 y 1996. Dado que la causa central del colapso económico fue la aguda crisis del sector externo, el estudio considera, en segunda instancia, el derrumbe de las exportaciones, las importaciones y el deterioro de la cuenta corriente. La información estadística de esta sección permite establecer dos períodos claramente delimitados: el del colapso, 1990-1993 y el de la

\begin{tabular}{|lrrrrrrrrr|c|}
\hline \multicolumn{10}{|c|}{ CUADRO 1 } \\
& $\mathbf{1 9 9 0}$ & $\mathbf{1 9 9 1}$ & $\mathbf{1 9 9 2}$ & $\mathbf{1 9 9 3}$ & $\mathbf{1 9 9 4}$ & $\mathbf{1 9 9 5}$ & $\mathbf{1 9 9 6}$ & $\begin{array}{c}\text { Index 1990=1000 } \\
\mathbf{1 9 9 3}\end{array}$ & $\mathbf{1 9 9 6}$ \\
\hline $\begin{array}{l}\text { PIB (Ps m, precios } \\
\text { constantes de 1981) }\end{array}$ & 19.0 & 17.0 & 15.0 & 12.7 & 12.8 & 13.2 & 14.2 & 67 & 75 \\
$\%$ crecimiento & -3.0 & -10.5 & -11.8 & -15.0 & 0.7 & 2.5 & 7.8 & & \\
\hline
\end{tabular}

\section{CARACTERÍSTICAS DE LA RECUPERACIÓN}

En primer lugar, una nota general acerca de la información estadística. La que se usa en el presente estudio es 
recuperación hasta 1996. Esta información es suministrada por los indicadores de las columnas a la derecha que toman como año base $1990(1990=100)$.

\section{Producción interna}

Las cifras a partir de 1990 se dan en términos del PIB a precios constantes del año 1981. La notable disparidad entre la estructura de los precios externos e internos desde 1981 ha llevado a distorsiones en los cálculos del crecimiento real. No obstante, Cuba no es el único país que usa precios constantes de un año base relativamente distante (1981); Perú y Guatemala, por ejemplo, presentan un problema similar. De otra parte, no hay indicación alguna de que se esté considerando la modificación del año base, al menos en el futuro inmediato.

Una limitación adicional alrededor de estas cifras, es que ellas sólo cubren el sector formal. La aguda contracción del PIB, combinada con los precios fijos, proveen un ambiente ideal para el surgimiento de actividades de mercado negro, las cuales indudablemente han incrementado su participación en la actividad económica entre 1990 y 1993. Los márgenes de ganancia en el mercado negro y el sector informal se han reducido, pero su infraestructura se ha consolidado. El rompimiento gradual y desmantelamiento de este sector, a medida que se logra la restauración macroeconómica y los desbalances se reducen, aparecen como un objetivo central de la política económica. Un estudio de la magnitud y la estructura de este sector sería de gran interés; pero este documento, como es habitual con los estudios macroeconómicos, se circunscribe al análisis del sector formal.

En el cuadro 2 PIB por sector y empleo podemos observar lo siguiente. La economía se contrajo en un $33 \%$ entre 1990 y 1993 y creció el $12 \%$ entre 1993 y 1996 . En 1996, el PIB permanecía 25\% debajo de su nivel en 1990. En términos porcentuales el sector más afectado por la contracción económica fue el de la construcción, seguido por el sector agrícola, en el cual la producción fue reducida a la mitad entre 1990 y 1994. En términos globales, las bajas más importantes se presentaron en el sector agrícola y manufacturero. En sólo un sector, el sector de minas, el incremento ha superado su nivel de 1989. Los servicios comunitarios, sociales y personales no se vieron afectados por la crisis, lo que refleja claramente la política gubernamental. Como porcentaje del PIB, éstos se incrementaron del $20 \%$ en 1990 al $29 \%$ en 1993 para caer de nuevo al $25 \%$ en 1996.

El sector de comercio, restaurantes y hoteles es casi tres veces mayor que el sector de la agricultura, en términos de participación en el PBI; y sólo se ha recuperado en dos terceras partes en relación a su nivel de 1989. Este sector se encuentra muy vagamente definido, por lo

\begin{tabular}{|c|c|c|c|c|c|c|c|c|c|}
\hline \multicolumn{10}{|c|}{$\begin{array}{l}\text { CUADRO 2: PIB POR SECTOR Y EMPLEO TOTAL } \\
\text { (Ps m, precios constantes de 1981) }\end{array}$} \\
\hline & \multirow[t]{2}{*}{1990} & \multirow[t]{2}{*}{1991} & \multirow[t]{2}{*}{1992} & \multirow[t]{2}{*}{1993} & \multirow[t]{2}{*}{1994} & \multirow[t]{2}{*}{1995} & \multirow[t]{2}{*}{1996} & \multicolumn{2}{|c|}{ Index $1990=1000$} \\
\hline & & & & & & & & 1993 & 1996 \\
\hline $\begin{array}{l}\text { Comercio, restaurantes } \\
\text { y hoteles }\end{array}$ & 4936 & 4396 & 4050 & 2936 & 2935 & 2985 & 3251 & 59 & 66 \\
\hline Manufacturas & 4640 & 4200 & 3507 & 3104 & 3341 & 3555 & 3835 & 67 & 83 \\
\hline $\begin{array}{l}\text { Servicios sociales } \\
\text { y personales }\end{array}$ & 3816 & 3753 & 3713 & 3748 & 3681 & 3548 & 3611 & 98 & 95 \\
\hline Agricultura y pesca & 1756 & 1335 & 1197 & 925 & 879 & 915 & 1075 & 53 & 61 \\
\hline $\begin{array}{l}\text { Transporte } \\
\text { y comunicación }\end{array}$ & 1202 & 1059 & 912 & 733 & 709 & 748 & 813 & 61 & 68 \\
\hline Construcción & 1508 & 1085 & 604 & 386 & 384 & 412 & 539 & 26 & 36 \\
\hline $\begin{array}{l}\text { Finanzas,valores } \\
\text { mobiliarios e } \\
\text { inmobiliarios }\end{array}$ & 603 & 639 & 544 & 513 & 492 & 484 & 519 & 85 & 86 \\
\hline $\begin{array}{l}\text { Gas, electricidad } \\
\text { y agua }\end{array}$ & 455 & 427 & 378 & 335 & 350 & 384 & 398 & 74 & 88 \\
\hline Minería & 92 & 82 & 106 & 96 & 98 & 152 & 177 & 106 & 194 \\
\hline PIB & 19008 & 16976 & 15010 & 12777 & 12868 & 13184 & 14218 & 67 & 75 \\
\hline $\begin{array}{l}\text { Puestos de trabajo } \\
\text { (en miles) }\end{array}$ & 4394 & 4374 & 4352 & 4313 & 4195 & 4131 & 4240 & 98 & 96 \\
\hline
\end{tabular}




\begin{tabular}{|c|c|c|c|c|c|c|c|c|c|}
\hline \multicolumn{10}{|c|}{$\begin{array}{l}\text { CUADRO 3: EXPORTACIONES DE BIENES } \\
\text { (Ps m) }\end{array}$} \\
\hline & \multirow[t]{2}{*}{1990} & \multirow[t]{2}{*}{1991} & \multirow[t]{2}{*}{1992} & \multirow[t]{2}{*}{1993} & \multirow[t]{2}{*}{1994} & \multirow[t]{2}{*}{1995} & \multirow[t]{2}{*}{1996} & \multicolumn{2}{|c|}{ Index $1990=1000$} \\
\hline & & & & & & & & 1993 & 1996 \\
\hline Azúcar & 4343 & 2291 & 1243 & 720 & 600 & 714 & 971 & 17 & 22 \\
\hline Pesca & 99 & 125 & 105 & 70 & 80 & 123 & 126 & 71 & 127 \\
\hline Minerales & 400 & 240 & 235 & 160 & 165 & 333 & 424 & 40 & 106 \\
\hline Tabaco & 114 & 114 & 94 & 70 & 70 & 101 & 126 & 61 & 111 \\
\hline Otros & 459 & 192 & 107 & 117 & 466 & 237 & 203 & 25 & 44 \\
\hline Total & 5415 & 2962 & 1784 & 1137 & 1381 & 1507 & 1850 & 21 & 34 \\
\hline
\end{tabular}

cual se presentan dificultades para realizar un análisis claro del mismo.

Mientras en 1993, la producción total fue un $33 \%$ por debajo de su nivel en 1990; la disminución del empleo se estimó en un $2 \%$ para el mismo período. Sin embargo, desde 1993 el empleo se ha reducido en un $2 \%$ mientras la economía creció en un $12 \%$. Esto refleja la política de retención de la fuerza laboral hasta que se puedan hallar fuentes alternativas de empleo. Una consecuencia de esto ha sido el decaímiento drástico de la productividad promedio hasta 1993, año a partir del cual se ha observado una recuperación aunque lenta. Este porcentaje encierra considerables divergencias entre las diferentes áreas productivas dado que la racionalización se ha introducido sector por sector.

\section{El sector externo}

Todas las cifras se suministran en pesos cubanos y a precios actuales. Dado que la tasa oficial de cambio es \$1:US\$1, éstas son de hecho valores en dólares.
En el cuadro 3 exportaciones de bienes podemos ver que:

Entre 1993 y 1996 se produjo un incremento del 63\% en las exportaciones. Aun así, en 1996 el total de bienes de exportación, equivalía a sólo la tercera parte de su nivel en 1990. Las exportaciones están poco diversificadas. El sector azucarero representó el $80 \%$ de los bienes de exportación en 1990. En 1993 se redujo al 63\% y en 1996 cayó aún más y su nivel fue del $52 \%$.

Las divisas por exportaciones del sector azucarero cayeron a su nivel más bajo en 1994, cuando representaron sólo un 14\% del nivel alcanzado en 1990 . Su recuperación hasta 1996 fue casi del $60 \%$ en relación a 1994 . No obstante, la cifra es aún un $20 \%$ menor que la de 1990 .

La diversificación de las exportaciones desde 1990 han tenido lugar solamente en tres actividades del sector primario: pesca, minerales y tabaco. Estos representaron el $11 \%$ de las exportaciones en 1990 , el $26 \%$ en 1993 y el $37 \%$ en 1996 . Mientras la pesca, los minerales y el tabaco aumentaron sus ingresos comparados con los de 1990; la suma total del resto de las exportaciones se mantiene por

\begin{tabular}{|lrrrrrrrrrr|}
\hline \multicolumn{7}{|c|}{ CUADRO 4: IMPORTACIÓN DE BIENES } \\
(Ps m)
\end{tabular}


debajo del 50\% de su nivel de 1990. Sin embargo, algunas de las diferencias entre el sector no-azucarero y el denominado "otros" podría explicarse por las modificaciones introducidas en el método usado para calcular el crecimiento del sector minero. Esto permite concluir que todavía no se ha dado un progreso significativo en el crecimiento de las exportaciones no-tradicionales.

En el Cuadro 4 importación de bienes podemos ver que las importaciones de bienes se contrajeron en un 73\% entre 1990 y 1993 y de 1993 a 1996 se incrementaron en un $74 \%$. No obstante, aún se mantienen por debajo del $50 \%$ de su nivel en 1990.

Debido al colapso económico, la participación de combustibles y alimentos en las importaciones totales se incrementó de $27 \%$ y $12 \%$ en 1990 a $37 \%$ y $26 \%$ en 1993 respectivamente. En 1996 esta participación se redujo a un $30 \%$ y $21 \%$ respectivamente. Las importaciones de combustible se mantienen en 52\% del nivel registrado en 1990, mientras el PIB recuperó hasta alcanzar 75\% de su nivel en 1990. Aunque la eficiencia en el uso de energía ha mejorado, la escasez de combustibles se ha constituido en un serio freno a la producción.

El rápido crecimiento de la importación de alimentos desde 1994, ilustra claramente el hecho de que las iniciativas políticas actuales no han logrado obtener éxito en el esperado incremento de la producción interna de alimentos.
Para 1994 las importaciones de maquinaria y equipos habían descendido drásticamente a solo $5 \%$ de su nivel en 1990. En 1996, éstas aún equivalían a solo la quinta parte de su nivel en 1990. De ahí el énfasis que se le ha dado al desarrollo de la industria de piezas de repuesto durante el período especial.

La categoría que creció más rápido, aunque el nivel de referencia con el que se le compara es bajo, es la categoría "otros". Parte de este incremento fue debido a las importaciones de bienes de consumo para los almanecenes regidos por el dólar; los gastos en esta categoría parecen mantener una relación positiva con las remesas reportadas en el balance de la cuenta corriente.

El cuadro 5 cuenta corriente muestra que el déficit comercial se redujó a $\$ 300$ millones entre 1990 y 1992; desde entonces se ha incrementado hasta alcanzar un nivel superior al presentado en 1990. El aumento de las divisas proveniente del sector turismo así como las remesas han contribuido a cubrir el amplio déficit comercial.

El crecimiento del turismo ha sido notable, ayudado por la enorme sub-utilización de recursos naturales en Cuba, lo que ha permitido obtener altos niveles de rendimiento con bajos niveles de inversión de capital. No obstante, sus recursos no son ilimitados y se espera que en el futuro el crecimiento del sector sea más moderado.

Los ingresos netos por remesas son mayores que los ingresos netos por turismo. Esto se explica porque en el

\begin{tabular}{|c|c|c|c|c|c|c|c|c|c|}
\hline \multicolumn{10}{|c|}{$\begin{array}{l}\text { CUADRO 5: CUENTA CORRIENTE } \\
\text { (Ps m) }\end{array}$} \\
\hline & \multirow[t]{2}{*}{1990} & \multirow[t]{2}{*}{1991} & \multirow[t]{2}{*}{1992} & \multirow[t]{2}{*}{1993} & \multirow[t]{2}{*}{1994} & \multirow[t]{2}{*}{1995} & \multirow[t]{2}{*}{1996} & \multicolumn{2}{|c|}{ Index $1990=1000$} \\
\hline & & & & & & & & 1993 & 1996 \\
\hline $\begin{array}{l}\text { Exportación } \\
\text { de bienes }\end{array}$ & 5415 & 2962 & 1784 & 1137 & 1381 & 1507 & 1850 & 21 & 34 \\
\hline $\begin{array}{l}\text { Importación } \\
\text { de bienes }-7417\end{array}$ & -4234 & -2315 & -1984 & -2353 & -2992 & -3610 & 27 & 49 & \\
\hline Balance de bienes & -2001 & -1272 & -531 & -847 & -971 & -1484 & -1761 & - & \\
\hline $\begin{array}{l}\text { Entrada de servicios } \\
\text { y rentas }\end{array}$ & 540 & 598 & 58 & 854 & 1171 & 1428 & 1985 & 158 & 368 \\
\hline de las cuales turismo & 243 & 387 & 567 & 756 & 850 & 1100 & 1350 & 311 & 555 \\
\hline $\begin{array}{l}\text { Salida de servicios } \\
\text { y rentas }\end{array}$ & $-500^{a}$ & $-500^{a}$ & -575 & -641 & -931 & -1108 & -1105 & 128 & 221 \\
\hline $\begin{array}{l}\text { Balance de servicios } \\
\text { y rentas }\end{array}$ & 40 & 98 & 183 & 213 & 241 & 320 & 880 & - & - \\
\hline Transferencias netas & -13 & 18 & 43 & 263 & 470 & 646 & 744 & & \\
\hline $\begin{array}{l}\text { Balance de cuenta } \\
\text { corriente }\end{array}$ & $-1974,3$ & $-1156,3$ & $-304,9$ & $-371,4$ & $-260,4$ & $-517,7$ & $-137,2$ & - & - \\
\hline$\%$ de PIB & 9.9 & 6.2 & 1.8 & 2.5 & 1.7 & 3.2 & 0.8 & & \\
\hline
\end{tabular}

${ }^{a}$ EIU estimate 
caso de este último un $60 \%$ de las ganancias obtenidas se utilizan para importaciones que cubren necesidades del mismo sector.

Sin embargo, establecer una clara diferencia entre las divisas provenientes de remesas y las de turismo es difícil. Parte del incremento en las remesas probablemente obedece al gasto de los turistas en el sector informal (o a las transferencias corrientes hechas a los cubanos).

El déficit de la cuenta corriente disminuyó rápidamente desde 1990, como resultado de la cancelación de los créditos del CAME. El déficit es relativamente pequeño. La carencia de acceso al sistema financiero internacional significa que el déficit de la cuenta corriente se mantendrá a niveles modestos, aunque la importación de bienes de capital comience a recuperarse.

No hay datos oficiales del PIB en dólares. Nuestras estimaciones del déficit de cuenta corriente se basan en nuestro propio cálculo aproximativo del PIB en dólares.

\section{LIMITACIONES}

\section{Limitaciones Externas al Crecimiento Económico}

El colapso económico de principios de los noventa se originó en un shock externo que llevó no solo a la pérdida de los mercados del CAME, sino también a la desaparición de las fuentes de financiación provenientes de estos países. La dependencia de Cuba del comercio internacional es considerable. En 1990, la proporción del comercio (exportaciones e importaciones de bienes y servicios) como porcentaje del PIB fue de casi el 70\%. El sector externo ha desempeñado un papel muy importante en la recesión económica, que se refleja en el descenso de la proporción entre comercio y el PIB que estuvo por debajo del 30\% en 1993 (el año más álgido de la crisis). La alta participación de alimentos y combustibles en las importaciones totales de 1996 demuestra la importancia de recuperar la capacidad de importación del país a fin de reestablecer un crecimiento económico sostenido. Por ello, el crecimiento de las exportaciones, el acceso a nuevos mercados y la obtención de nuevos recursos financieros determinarán el desempeño de la economía en los próximos años.

Las posibilidades de expandir el comercio y por consiguiente la recuperación de la economía en su conjunto, dependen de dos factores: el sector azucarero y el potencial para la diversificación. La disminución en la producción del sector azucarero ha sido el factor determinante en la caída de las exportaciones. Esto se explica por la caída de la producción así como por la desaparición de los precios subsidiados ofrecidos por el CAME. Aun si la producción alcanzara los volúmenes de 1990, con los actuales bajos precios del azúcar en el mercado internacional, su valor en dólares equivaldría sólo a la tercera parte de los ingresos obtenidos en ese año. No está claro cual es el nivel de producción de azúcar económicamente eficiente para Cuba, ya que los precios internos no se toman en consideración cuando se calculan rendimientos. Expertos de la industria azucarera han sugerido que el país debe producir entre 5 y 6 millones de toneladas anuales, en comparación con los 7.8 millones producidos en la cosecha de 1990-91. Segun la tendencia de los precios mundiales, esto aún dejaría un vacío por encima de $\$ 2 \mathrm{~m}$ en el balance comercial con respecto a los ingresos de 1990 . La recuperación del sector azucarero será por ello parte fundamental en el restablecimiento de la capacidad importadora. Sin embargo su mera recuperación no será condición suficiente.

El sector azucarero ha sido reorganizado desde 1993, pero los resultados no han sido los esperados.

\section{Producciones, exportaciones y ganancias del sector azucarero}

Parte del déficit comercial puede ser cubierto por el turismo y las remesas. Cuba tiene la gran ventaja de poseer recursos naturales que le dan una ventaja comparativa para el turismo y además cuenta con una gran comunidad exiliada, que envía considerable cantidad de dinero para apoyar a sus familias. Pero los ingresos del turismo y de las remesas pueden ser volátiles, y la dependencia de estos flujos además de suponer un riesgo es una fuente de conflicto social. El monto total de los ingresos por turismo, ha crecido desde 1990 y se espera que su crecimiento en el futuro sea más lento a medida que la industria hace más uso de su capacidad subutilizada. Los estímulos producidos por el turismo podrían tener un impacto mayor, si estos contribuyen a incrementar la producción interna y se subtituyen las importaciones

$\mathrm{El}$ inconveniente con las remesas es que incrementan la tendencia a la importación. La correlación entre éstos y el nivel de importaciones de bienes para consumo privado lo demuestra. El desarrollo del sector financiero podría ayudar a canalizarlas hacia el incremento del ahorro interno en un futuro cercano.

Se ha producido un progreso sustancial en los sectores de minas, pesca y tabaco y se estima que a medio plazo éstos posean la capacidad para mantener su rápido nivel de crecimiento, dado que la financiación -incluyendo crédito externo- es asequible y que pueden ser obtenidos nuevos mercados de exportación. Sin embargo, estos tres sectores no serían suficientes para compensar el vacío dejado por la baja en las exportaciones de los bienes no azucareros desde 1990 . Por lo tanto, una mayor diversificación en las exportaciones ayudaría a lograr un crecimiento sostenido.

Al comienzo del período especial se consideró que los productos farmacéuticos ofrecían un gran potencial de crecimiento, pero las expectativas no han fructificado. Parece que las barreras proteccionistas establecidas por las multinacionales del sector en contra de nuevos productores se han convertido en un obstáculo mayor de lo esperado. Algunos contratos de exportación se han concretado, y se espera que los ingresos del sector crezcan con el tiempo, pero es probable que la contribución del sector farmacéutico no sea de la magnitud que se esperaba originalmente. 
Las exportaciones de otros bienes manufacturados no han progresado mucho. Un prerequisito para incrementar las exportaciones es el establecimiento de acuerdos de comercio bilateral y acuerdos de comercialización con compañías extranjeras. En esto se ha trabajado arduamente. Debe tenerse en cuenta que la mayor parte de la planta manufacturera cubana es heredera del CAME y por este hecho es incompatible con los requerimentos del nuevo mercado mundial o simplemente son obsoletos. Sin una fuerte inversión de capital en el sector manufacturero no se puede esperar un mejoramiento en su contribución a las exportaciones en un futuro próximo.

Un inconveniente más es que los niveles salariales no pueden competir con los de sus vecinos en la región; esto es, si la garantía del nivel de vida, el cual es una prioridad central de la estrategia del desarrollo, aspira a ser mantenido. El éxito dependerá de la habilidad para lograr niveles de productividad suficientemente altos que hagan al sector laboral más competitivo. El nivel de educación relativamente alto y las capacidades laborales de la fuerza laboral cubana proveen el potencial para realizar esto. Para aprovechar estos recursos es necesario encontrar fuentes de inversión, tecnología y mercados. Hasta ahora se han hecho muchos anuncios de negociaciones que estarían en progreso y se ha sugerido que existe una amplia gama de contratos firmados; sin embargo, los ingresos devengados no son evidentes todavía. El levantamiento de las sanciones comerciales estadounidenses serían una gran contribución a la potencialidad comercial. Sin embargo, esto no se dará en el corto plazo.

\section{El problema financiero}

Una caída en las exportaciones en el nivel experimentado por Cuba vendría acompañado normalmente por un amplio déficit en la cuenta corriente. Pero en el caso de Cuba, esto no se produjo. Cuba se vió forzada entonces a recuperarse de este colapso con los escasos y muy limitados recursos que ofrecía el sistema financiero internacional. El

\section{CUADRO 6.: FINANCIACIÓN EXTERNA (Ps m)}

\begin{tabular}{|lrrrr|}
\hline & $\mathbf{1 9 9 3}$ & $\mathbf{1 9 9 4}$ & $\mathbf{1 9 9 5}$ & $\mathbf{1 9 9 6}$ \\
\hline $\begin{array}{l}\text { Inversión externa } \\
\text { neta }\end{array}$ & 54 & 563 & 5 & 8 \\
$\begin{array}{l}\text { Otras inversiones } \\
\text { netas a largo plazo }\end{array}$ & 54 & 254 & 20 & 226 \\
$\begin{array}{l}\text { Flujos netos de } \\
\text { capital a corto plazo }\end{array}$ & 238 & -555 & 572 & -89 \\
$\begin{array}{l}\text { Variación en } \\
\text { las reservas }\end{array}$ & 16 & -2 & -79 & -8 \\
Total & 372 & $\mathbf{2 6 0}$ & $\mathbf{5 1 8}$ & $\mathbf{1 3 7}$ \\
\hline
\end{tabular}

acceso a esta fuente de finaciación fue obstruído por la legislación estadounidense y por la suspensión de pagos de la deuda externa desde 1986. De no haberse producido el estancamiento en las finanzas externas, la depresión económica no habría sido tan drástica y la necesidad de promover y desarrollar el turismo y reajustar la estructura de la economía, no hubieran sido tan urgentes.

Así, al tiempo que Cuba entraba en un proceso de recuperación, se le negaba el acceso a fuentes de capital externo que normalmente financian la inversión productiva y que hacen la recuperación económica sostenible. Dicha recuperación debe ser por ende más lenta de lo previsto. En lugar de experimentar un incremento a cargo de la deuda externa futura, Cuba se financia a través del pago de altas tasas de interés sobre los limitados créditos financieros obtenidos hasta el momento. Esto lo podemos observar en el cuadro 6 financiación externa aunque los datos son muy incompletos. No existen cifras del período entre 1990-93 y los mayores flujos se clasifican bajo la categoría "otros". Igualmente, las autoridades cubanas no proveen datos sobre las reservas internacionales. Sin embargo, dichos datos nos muestran que la poca discriminación de los flujos de inversión extranjera directa (IED) y las cifras para los flujos de las llamadas otras categorías sugieren que sólo unos pocos proyectos atrajeron esta inversión. Sobresalen los sectores de minas y telecomunicaciones.

El pequeño volúmen de flujos de IED refleja, bien la incapacidad para atraer capital extranjero, bien el poco entusiasmo gubernamental para brindar más oportunidades de inversión al capital extranjero o ambas cosas.

Las conversaciones sobre la deuda externa han sido difíciles. Las sanciones estadounidenses descartan la posibilidad de cualquier apoyo de parte del Fondo Monetario Internaciónal (FMI) y el Banco Mundial. Asimismo, la falta de transparencia en el reporte de cifras macroeconómicas es un obstáculo. Se espera que con el anuncio de la introducción de informes trimestrales sobre los datos macroeconómicos se presente una mejoría en este frente. Sin embargo, el nivel internacional de reservas continúa siendo considerado como un secreto de estado. La reestructuración del sector bancario ayudará a mejorar el nivel de los reportes económicos y a recuperar la confianza en las finanzas nacionales; pero esto llevará tiempo.

La normalización de las relaciones con los Estados Unidos sería de gran ayuda, pero esto no tendrá lugar en el corto plazo. La única manera rápida para garantizar el acceso al sistema internacional financiero sería adoptar un programa de privatización. Pero el gobierno cubano se opone totalmente a la venta de bienes estatales. El sector de telecomunicaciones es el único que ha avanzado en este proceso y hasta ahora se ha llevado a cabo una privatización parcial del mismo. Pero esto parece ser más un proceso de capitalización que una transferencia de la propiedad de los bienes. A pesar de que la ley de inversión extranjera de 1995 no prohibe la transferencia de bienes a extranjeros, especifica que se buscarán socios extranjeros sólo en la medida que estos aporten capital, nueva tecnología y mejo- 
ramiento en el manejo empresarial de los nuevos mercados. Hay contratos de riesgo en los sectores de minas y petróleo; contratos de concesión que se han ofrecido para proyectos menores en infraestructura; asimismo se han aprobado contratos para la construcción de condominios, pero no hay conocimiento de que propiedad alguna se haya cedido al capital extranjero. Dada la urgente necesidad de capital extranjero, la inflexible actitud del gobierno en este frente es notable.

Está claro que el programa masivo de privatización incrementaría el potencial de recuperación económica. Una razón por la cual esto podría generar dificultades de llevarse a cabo, sería el problema de establecer derechos de propiedad sobre estos bienes. La mayoría de las empresas provisoras de servicios públicos, áreas de cultivo y empresas manufactureras que funcionaban desde antes de 1959 están sujetas a demandas en juzgados norteamericanos por parte de los expropiados (estadounidenses o exiliados cubanos). Sin embargo, aún si esto pudiese obviarse, un programa de privatización acelerado crearía otros problemas adicionales: el estado cubano se vería incapacitado para obtener buenos precios para los bienes en venta, porque muy posiblemente los compradores tratarían de tomar ventaja de la situación, así como de las dificultades para obtener una adecuada evaluación de los bienes y de la ausencia de competidores estadounidenses. La ausencia de un mercado financiero nacional, haría que la comercialización interna de los bienes fuera imposible. Asimismo la poca acumulación de riqueza privada desde 1959 significaría que todos los inversionistas serían extranjeros. Además, el sistema de manejo empresarial centralizado ha legado una estructura de abastecimiento de caracter monopólico.
Dados estos obstáculos, se puede entender mejor porque que el gobierno ha elegido la actual estrategia (un proceso de reforma lento).

La creación de un mercado financiero está teniendo lugar, con un programa nacional intensivo de entrenamiento en manejo empresarial y principios bancarios. Las empresas están siendo reestructuradas como unidades autónomas, una por una; como parte de este programa nacional muchas han sido divididas en pequenas unidades. Formas de propiedad alternativa a la estatal están siendo también consideradas. Las unidades básicas de producción cooperativas (UBPC) aportaron un programa piloto para la agricultura, pero sin mucho éxito. Sin embargo, éste ha sido importante como experiencia de aprendizaje. Las empresas de las Fuerzas Armadas y la industria turística han encabezado este proceso de experimentación y restauración donde quiera que éste se ha producido. Los sectores del acero, los textiles y la pesca han sido reestructurados. Este programa de reestructuración planificada es un intento para introducir reformas que en otros sectores han sido adelantadas por el mercado pero antes de que el mercado llegue. Esto muestra como el sistema económico se está preparando para enfrentar la llegada masiva del capital extranjero. Pero hasta que esto ocurra la inversión directa consistirá solo en un reducido número de acuerdos que carecen de sistematicidad.

El establecimiento reciente de la zona de libre comercio, es un intento más para atraer a los inversionistas al sector manufacturero que se dedica a la exportación; es una forma de obviar la incertidumbre que ronda alrededor de la disputa de los bienes de empresas ya establecidas. Las nuevas negociaciones no sólo proveeran emple-

CUADRO 7: ESTADOS CONTABLES

(Ps $\mathrm{m}$, precios corrientes)

\begin{tabular}{|lrrrrrrrrr|}
\hline & $\mathbf{1 9 9 0}$ & $\mathbf{1 9 9 1}$ & $\mathbf{1 9 9 2}$ & $\mathbf{1 9 9 3}$ & $\mathbf{1 9 9 4}$ & $\mathbf{1 9 9 5}$ & $\mathbf{1 9 9 6}$ & & \\
\hline Ingresos & $\mathbf{1 3 5 2 4}$ & $\mathbf{1 0 9 4 9}$ & $\mathbf{1 0 1 7 9}$ & $\mathbf{9 5 1 6}$ & $\mathbf{1 2 7 5 7}$ & $\mathbf{1 3 0 4 3}$ & $\mathbf{1 2 1 2 4}$ & $\mathbf{7 0}$ & $\mathbf{9 0}$ \\
Gastos & $\mathbf{1 5 4 8 2}$ & $\mathbf{1 4 7 1 4}$ & $\mathbf{1 5 0 4 8}$ & $\mathbf{1 4 5 6 7}$ & $\mathbf{1 4 1 7 8}$ & $\mathbf{1 3 8 0 9}$ & $\mathbf{1 2 6 9 2}$ & $\mathbf{9 4}$ & $\mathbf{8 2}$ \\
Gasto corriente & 11084 & 11089 & 11810 & 12529 & 11495 & 12064 & 10709 & 113 & 97 \\
Educación y salud & 2542 & 2429 & 24665 & 2462 & 2396 & 2498 & 2548 & 97 & 103 \\
Defensa y orden & & & & & & & & & \\
público & 1002 & 882 & 842 & 713 & 651 & 727 & 727 & 71 & 73 \\
Seguridad social & 1164 & 1226 & 1348 & 1452 & 1532 & 1573 & 1604 & 125 & 138 \\
Administración & 453 & 400 & 373 & 413 & 354 & 375 & 383 & 91 & 85 \\
Subsidios & 3981 & 4722 & 5300 & 6.168 & 4.884 & 4937 & 2886 & 155 & 72 \\
Otros gastos corrientes & 1942 & 1430 & 1482 & 1321 & 1678 & 1954 & 2561 & 68 & 132 \\
Gastos de capital & 4398 & 3625 & 3239 & 2038 & 2683 & 1745 & 1984 & 46 & 45 \\
Balance sector público & $-\mathbf{1 9 5 8}$ & $-\mathbf{3 7 6 5}$ & $\mathbf{- 4 8 6 9}$ & $\mathbf{- 5 0 5 1}$ & $\mathbf{- 1 4 2 1}$ & $\mathbf{- 7 6 6}$ & $\mathbf{- 5 6 8}$ & - & - \\
\% de PIB & $\mathbf{1 0 , 0}$ & $\mathbf{2 3 , 2}$ & $\mathbf{3 2 , 7}$ & $\mathbf{3 3 , 5}$ & $\mathbf{7 , 8}$ & $\mathbf{3 , 6}$ & $\mathbf{2 , 4}$ & - & - \\
\hline
\end{tabular}


os, sino que también darán una oportunidad a los gestores de la política económica para experimentar con las recientes estructuras regulativas, en comparación con las prácticas del sistema económico centralizado que ha regido el país durante décadas.

\section{Restricciones internas al crecimiento}

Dados los limitados recursos externos se entiende que el crecimiento sólo podría generarse a través de recursos internos; esto es, incrementando la inversión doméstica y mejorando la eficiencia económica.

El nivel de inversión pública es limitado debido a las restricciones fiscales como podemos observar en el cuadro 7 estados contables.

Desde 1993 el gobierno ha buscado restaurar el equilibrio macroeconómico mediante la recuperación del balance fiscal. El déficit del sector público ha sido reducido de un 34\% del PIB en 1993 a un $2.4 \%$ en 1996. Los ingresos fiscales han sido incrementados a través del alza en los productos secundarios de la canasta básica y por medio de la introducción de nuevos impuestos al consumidor.

El presupuesto para la salud, la educación y la seguridad social, ha sido sostenido casi en su nivel a pesar de la crisis, mientras que las reducciones más significativas fueron hechas en la defensa y en el gasto del gobierno central.

Los recursos del presupuesto para las inversiones a largo plazo cayeron de Ps4.4 billones en 1990 a Ps2 billones en 1993 y se ha mantenido en este nivel desde entonces.

Para incrementar el volumen de inversión pública, se requiere un incremento en los ingresos fiscales y una reducción de los subsidios.

La oficina nacional de impuestos tiene centrada ahora su atención en diseñar un nuevo sistema de impuestos que se acomode a una economía mixta. A pesar de algunas dificultades los logros en este frente han sido positivos. Los datos recopilados demuestran que se ha realizado una ardua labor en materia de capacitación y en general el desempeño de los recolectores e impuestos ha sido bueno. Sin embargo, el trabajo por hacerse es enorme dado que el sistema apenas da sus primeros pasos, especialmente en unas condiciones nuevas donde el crecimiento del sector informal constituye un gran reto.

La reducción de subsidios, en una economía que funciona por debajo de su capacidad, inevitablemente lleva al despido de un considerable número de empleados. Pero la celeridad de este proceso está limitado por el compromiso del gobierno de proveer fuentes alternativas de empleo a aquellos que lo pierdan. Esto hace más lento el proceso de reestructuración y significa que la parte subsidios en el presupuesto nacional incluye un elemento que bien podría ser descrito como costos de seguridad social.

La reducción de los subsidios a la agricultura es también problemático desde el punto de vista político y social; sin embargo, los fallos del sector están siendo analizados cuidadosamente. Aunque las dificultades de la industria azucarera se deben principalmente a la carencia de recursos financieros para la obtención de insumos esenciales para la producción; también se han identificado ineficiencias como un problema central. Las implicaciones del reciente reemplazo del ministro de este sector por un general de las Fuerzas Armadas parece ser un signo de racionalización de la industria. En el sector alimenticio los diversos intentos para mejorar la producción y distribución no han obtenido los resultados esperados. La obtención de alimentos en los mercados libres agropecuarios parece haber aumentado, sin embargo, la producción de alimentos para los abastecedores estatales (manejado con precios fijos) continúa siendo estática.

El continuo uso de cuotas y precios estatales se ha convertido en la dificultad primordial. Cabe decir que ya se han tomado medidas para dar paso a la liberación de los antes mencionados. Para dicha liberación se ha tomado el precio del combustible (en dólares) como programa piloto, el cual se aspira a introducir de manera generalizada para la agricultura de exportación. La provisión de raciones con un amplio subsidio en los precios es una de las obligaciones fiscales más costosas, los costos aumentarían aún más si el estado paga el precio fijado por el mercado para las cuotas que él recibe de los agricultores. El sistema de ración es otro de los pilares primordiales del sistema de la seguridad social. Es probable que la producción de alimentos no mejore significativamente hasta que los inconvenientes del sistema no se resuelvan. Es decir, hasta que el Estado brinde más incentivos a los agricultores.

\section{CONCLUSIONES: PERSPECTIVAS PARA LA RECUPERACIÓN}

Con las reformas introducidas al manejo del sistema económico y las limitaciones que presenta el sector externo, el crecimiento podría continuar durante algunos años más, ayudado por la expansión del turismo y el incremento de remesas. Pero éste no sólo sería un crecimiento frágil, sino que entraría en un proceso de estancamiento en unos pocos años. Se incrementaría el desempleo abierto y se presentaría una gran expansión del mercado negro. Pero como ya se ha argumentado, algunas limitaciones no son insalvables o por lo menos podrían ser menos inflexibles en un futuro cercano a condición de que se prosiga con el proceso de transición o "restructuración".

El desarrollo de nuevas iniciativas para el manejo empresarial, estrechamente ligado con el establecemiento de un mercado financiero, transformará radicalmente el rol de la planeación económica y del mercado. La reformas al sistema de precios y la privatización están aún distantes, pero se están sentando las condiciones para que el proceso sea lo menos traumático posible. De ahí la posibilidad de que, a pesar de la muy precaria situación financiera bajo la cual trabaja el gobierno cubano, exista una salida a la crisis que lleve a un desarrollo económico sostenido. Pero en la búsqueda de este nuevo camino se requiere la derrota de muchos tabús en tanto que las dificultades económicas persistirán. El crecimiento puede ser generalmente positivo, aunque un poco desbalanceado (como en 1996-97), como resultado de la carencia de divisas internacionales. 


\section{RESUMEN}

Este artículo trata de explicar la recuperación de la economía cubana en el período 1993-1996, presentando la estructura de la producción y el comercio entre 1990 y 1996, y analizando las limitaciones tanto de carácter doméstico como externo para su mantenimiento. Finalmente, la autora aventura algunas proyecciones de esta economía para los próximos años.

Palabras claves: recuperación económica, estructura de la producción, comercio, política económica.

\section{ABSTRACT}

This article aims at explaining the recovery of the Cuban economy in the 1993-96 period. It describes the structure of economic production and commerce from 1990 to 1996, and analyzes domestic and international limits for the persistence of the Cuban economic structure. It also discusses the future prospects of the Cuban economy.

Key words: economic recovery, structure of production, commerce, economic policy.

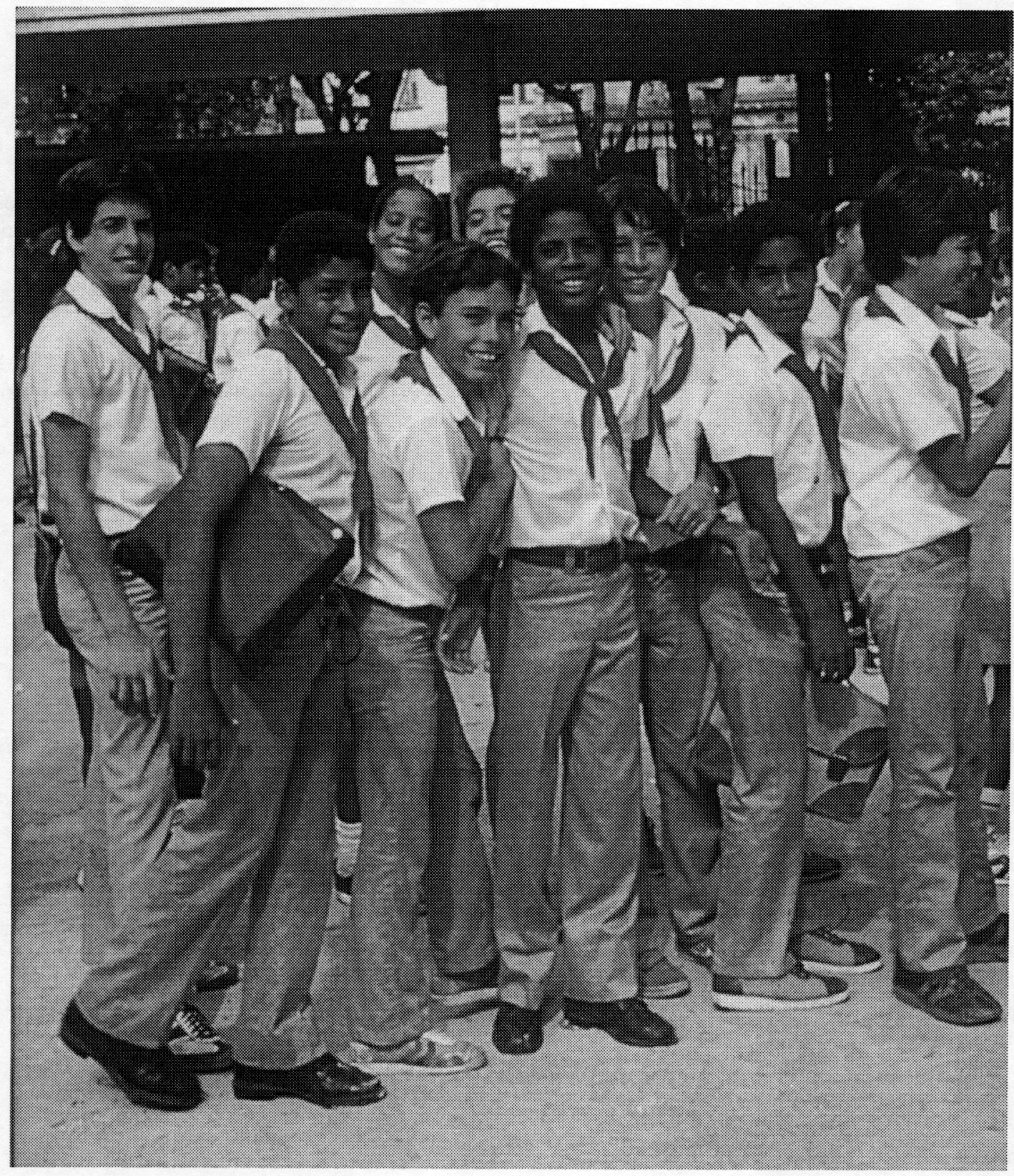

